\title{
DEMAND FORECASTING FOR MOTOR VEHICLE SPARE PARTS
}

\author{
J J Strasheim Pr Eng \\ Department of Industrial and Systems Engineering \\ University of Pretoria
}

\section{ABSTRACT}

Reordering motor vehicle spare parts for the purposes of stock replenishment is an important function of the parts manager in the typical motor dealership. Meaningful reordering requires a reliable forecast of the future demand for items. A variety of alternative forecasting techniques were evaluated for this purpose with the aim of selecting one optimal technique to be implemented in an automatic reordering module of a real time computerized inventory management system.

\section{OPSOMMING}

Aanvulling van onderdeelvoorraad is 'n belangrike funksie van die onderdelebestuurder in die tipiese motorhandelsaak. Sinvolle herbestelling vereis 'n betroubare vooruitskatting van die toekomstige vraag na items. 'n Verskeidenheid alternatiewe vooruitskattingstegnieke is evalueer ten einde 'n optimale tegtniek te vind vir implementering in die outomatiese herbestelmodule van ' $n$ intydse gerekenariseerde voorraadbestuurstelsel. 


\section{INTRODUCTION}

Although there are many forecasting techniques and the literature sources on the subject are extensive, there seems to be an absence of literature on specifically forecasting demand for motor vehicle spare parts in the retail sector. The reason for this is probably that it is not the easiest application of forecasting theory probably due to low sales volumes, high numbers of stock items, very restrictive practical requirements and irregular demand patterns.

In general, any forecasting technique is required to be accurate, have low cost of application as well as be easy to use and maintain in practice for it to be an optimal choice. The specific circumstances in the motor industry requires some additional features from such a technique. It is also required to forecast three months demand per item due to reordering policies and lead times. It should not require more than 12 months demand history (ideally only 6 months) to produce good results, as the most computer systems only carry 12 months demand history. It must also produce reasonable results with little history so that reliable forecasts for new items in the database as well as new model parts can be quickly attained. Computation needs to be fast and effective as the average stock system contains anything between 6,000 and 26,000 line items and if calculations are extensive and complex it can take a very long time for a reorder activity.

\section{ALTERNATIVE TECHNIQUES}

\subsection{GENERAL}

A large number of forecasting techniques were researched and evaluated, namely :

Simple Averaging

Averages, Moving Averages, Double Moving Averages.

\section{Exponential Smoothing}

Single Exponential Smoothing, Adaptive Exponential Smoothing, Double Exponential Smoothing (Brown's one parameter linear method and Holt's two parameter method), Triple Exponential Smoothing (Brown's one parameter quadratic method and Winter's three parameter trend and seasonality method).

\section{Other Smoothing Methods}

Chow's Adaptive Smoothing, Brown's One Parameter Adaptive Smoothing, Harrison's Harmonic Smoothing.

Regression

Linear Regression, Multiple Regression.

\section{Decomposition}

Classical Decomposition, Census II.

Box-Jenkins ARIMA Time Series Methods.

Focus Forecasting. 


\subsubsection{AVERAGE.}

This is the most basic and most elementary of all forecasting techniques. It simply entails using the three times the average of all the available history as the forecast for the future three month period. It is cheap, fast and easy use and understand. It unfortunately does not recognize any trend or seasonal properties in the data which makes it unsuitable.

\subsubsection{MOVING AVERAGES.}

A moving average is one of the simplest forecasting techniques and is based on the assumption that historical data contains an element of noise which hides the real pattern of the time series. Moving averages may also be weighted to allocate more importance to the most recent observations. As soon as the data contains a meaningful trend component the moving average becomes less effective but it is cheap, fast and easy use and understand.

\subsubsection{DOUBLE MOVING AVERAGES.}

The double moving average is better in handling trend than the simple moving average. The technique is intuitively attractive, fast, easy and does not require lots of data to provide reasonable results.

\subsection{EXPONENTIAL SMOOTHING}

\subsubsection{SINGLE EXPONENTIAL SMOOTHING.}

This is one of the most used forecasting techniques and is in fact a special form of the weighted moving average. It allocates more weight to the most recent observations and allocates exponentially less weight to value as they get older based on a smoothing constant $\alpha$. The technique requires little storage and is easy to calculate. It unfortunately tends to underestimate when there is a downward trend in the time series and vice versa.

\subsubsection{ADAPTIVE SINGLE EXPONENTIAL SMOOTHING.}

The difference between this and the previous method is that the value of the smoothing constant $\alpha$ is dynamic and adapts according to changes in the time series. This method is generally described in literature as suitable in situations where forecasts have to be made for large numbers of items.

\subsubsection{DOUBLE EXPONENTIAL SMOOTHING - BROWN'S ONE PARAMETER LINEAR METHOD.}

What makes this method particularly attractive is the fact that it only requires 3 data values and a smoothing constant to make a forecast. It also allocates decreasing weights to the previous data values. Because moving averages lag the time series in the case of an underlying trend the difference between single and double smoothed values is added to the single smoothed value to result in a trend adjusted smoothed value. The calculation for this method is done as follows :

$$
\begin{array}{ll}
S^{\prime}(t) & =\alpha X(t)+(1-\alpha) S^{\prime}(t-1) \\
S^{\prime \prime}(t) & =\alpha S^{\prime}(t)+(1-\alpha) S^{\prime \prime}(t-1) \\
A(t) & =2 S^{\prime}(t)-S^{\prime \prime}(T)
\end{array}
$$




$$
\begin{gathered}
\text { http://sajie.journals.ac.za } \\
-21- \\
B(t)=(\alpha /(1-\alpha))\left(S^{\prime}(t)-S^{\prime \prime}(t)\right) \\
F(t+m)=A(t)+B(t) m
\end{gathered}
$$

where :

$$
\begin{array}{ll}
S^{\prime}(t) & =\text { Single exponentially smoothed value. } \\
S^{\prime \prime}(t) & =\text { Double exponentially smoothed value. } \\
t & =\text { Period number. } \\
m & =\text { Number of periods in the future to forecast. } \\
\alpha & =\text { Smoothing constant. }
\end{array}
$$

\subsubsection{DOUBLE EXPONENTIAL SMOOTHING - HOLTS TWO PARAMETER METHOD.}

Holt's method is to some extent the same as the previous method except that it smooths the trend value with a separate smoothing constant allowing for greater control.

\subsubsection{TRIPLE EXPONENTIAL SMOOTHING - BROWN'S ONE PARAMETER QUADRATIC METHOD.}

Just as double exponential smoothing is required when an underlying trend is linear, must higher order smoothing be used when the underlying trend is quadratic, cubic or even of a higher order. This approach of Brown's is based on a quadratic trend.

2.3.6 TRIPLE EXPONENTIAL SMOOTHING - WINTER'S THREE PARAMETER TREND AND SEASONALITYMETHOD.

All of the previous methods do not specifically provide for seasonal variations in the time series. Winter's method is based on three types of smoothing to specifically handle this shortcoming, namely overall smoothing, trend smoothing and seasonal smoothing, making it an attractive option. It has serious practical limitations though because of a seasonal cycle of 12 months being too long and the use of 3 smoothing constants pose a serious practical problem.

\subsection{OTHER SMOOTHING METHODS}

\subsubsection{CHOW'S ADAPTIVE SMOOTHING.}

This method is in principle the same as exponential smoothing with the only difference that it can also be used for non static time series.

\subsubsection{BROWN'S ONE PARAMETER ADAPTIVE SMOOTHING METHOD.}

It has been reported that this technique rendered acceptable results as far back as 1959 in practical situations. It requires one smoothing constant to determine the sensitivity of speed of reaction to changes in the time series.

\subsubsection{HARRISON'S HARMONIC SMOOTHING METHOD.}

This method, which requires knowledge of Fourier transformations, lots of data and many calculations is not suitable for the purpose of fast and simple forecasting. 


\subsection{REGRESSION}

\subsubsection{LINEAR REGRESSION.}

This technique is simple to understand and use and is based on fitting curves to the data with the method of least squares.

\subsubsection{MULTIPLEREGRESSION.}

The multiple regression model assumes that more than one independent variable determine the demand in the forecasted period. It is not an easy task to identify and maintain such variables in practice for large volumes of items.

\subsection{DECOMPOSITION METHODS}

This group of methods rely on the assumption that any time series consists of four underlying components, namely trend, seasonal, cyclical and random. It requires a fairly large number of observations to produce reasonable forecasts rendering it unusable.

\section{$2.7 \quad$ BOX-JENKINS METHODS}

This group of techniques are intuitively attractive but requires lots of data, requires substantial processing and is complex to understand.

\subsection{FOCUS FORECASTING}

Bernard Smith in his book "Focus Forecasting" [10] describes a method of forecasting that is somewhat new in its approach. It involves an approach where alternative simple forecasting strategies are evaluated for the immediate past period and selecting the best strategy for forecasting the next period.

\section{METHOD OF EVALUATION}

\subsection{GENERAL}

In order to evaluate all of the aforementioned techniques one has to use quantifiable measures. Forecasting theory describes many such measures, such as :

\section{Statistical measures}

Mean error, Mean absolute error, Sum of squared errors, Mean squared error, Standard deviation of errors.

\section{Relative measures}

Percentage error, Mean percentage error, Mean absolute percentage error.

\section{Other measures}

Theil's U-value, Durban-Watson value, Forecasting index. 
All of these measures apply nicely in a system where a single time series is being analyzed. They lose their effectiveness when forecasts have to be done for groups of items and large numbers of items. This is largely due to four factors.

o Firstly, the relative type of measure is more applicable than the absolute type of measure because the stock systems comprises many different items and one can not for example compare one spark plug to one engine subassembly.

- Secondly, none of the listed measures incorporate the value of items.

o Thirdly, the measures do not allow for the definition of what would be an acceptable forecast.

o Lastly, the measures sometimes involves some computational problems. For example, when confronted with a slow moving item, the forecast may be 1 and the actual demand 0 . What is the percentage error in this case ?

All of these factors necessitated the definition of a special mechanism whereby forecasts could be measured and compared.

\subsection{METHOD OF EVALUATION}

The following approach was adopted for evaluation of forecasts for a cumulative three month future period :

\section{Percentage Acceptable Forecast}

Only "Fast", "Medium" and "Slow" items were considered as these are the only items normally reordered on a bulk basis, where such items are classified according to frequency of demand.

A "percentage acceptable forecast" based on quantity was calculated, defined as follows : An error of between $-20 \%$ and $+10 \%$, or an absolute error of 1 out of 1 to 4 items, or an absolute error of 2 out of 5 to 8 items would be classified as acceptable.

The reasoning behind this method is that a larger minus error percentage is more acceptable that a large plus error because the stock can always be ordered again, but to get rid of excess stock is not quite as easy. Relaxing the allowable percentage error for low volumes of items was done to induce a more realistic acceptable percentage. One has to keep in mind that the forecasts are not blindly used to reorder, but are reviewed by a responsible person before finalizing an order.

\section{Percentage Cost Error}

The percentage cost error was expressed as the variance of the cumulative three month demand in terms of the actual value of demand in comparison to the forecasted value of demand.

Both measures were evaluated for all methods and were also separately evaluated for "Fast", "Medium" and "Slow" categories of items as well as for all items. 


\section{COMPARISON OF RESULTS}

The method that gave the best results most consistently for all categories of items and both measures of effectiveness was Brown's One Parameter Linear Method as described in paragraph 2.3.3.

The following tables summarize the results.

AVERAGE PERCENTAGE ACCEPTABLE FORECAST

\begin{tabular}{|l|l|l|}
\hline & Average & Range \\
\hline All items $(\alpha 0.10)$ & 57.3 & 10.1 \\
\hline Fast items $(\alpha 0.00)$ & 47.9 & 5.3 \\
\hline Medium items $(\alpha 0.10)$ & 62.2 & 6.5 \\
\hline Slow items $(\alpha 0.10)$ & 71.8 & 7.5 \\
\hline
\end{tabular}

\section{COST VARIANCE PERCENTAGE}

\begin{tabular}{|l|l|l|}
\hline & Average & Range \\
\hline All items $(\alpha 0.70)$ & 0.3 & 60.1 \\
\hline Fast items $(\alpha 0.80)$ & 0.7 & 69.9 \\
\hline Medium items $(\alpha 0.40)$ & 2.7 & 35.2 \\
\hline Slow items $(\alpha 0.70)$ & 3.6 & 49.9 \\
\hline
\end{tabular}

\section{APPLICATION}

A comparison of the actual versus forecast for the cumulative three month demand for typical "Fast" items was performed using Brown's one parameter linear method.

The results are shown in figures 1,2 and 3. 


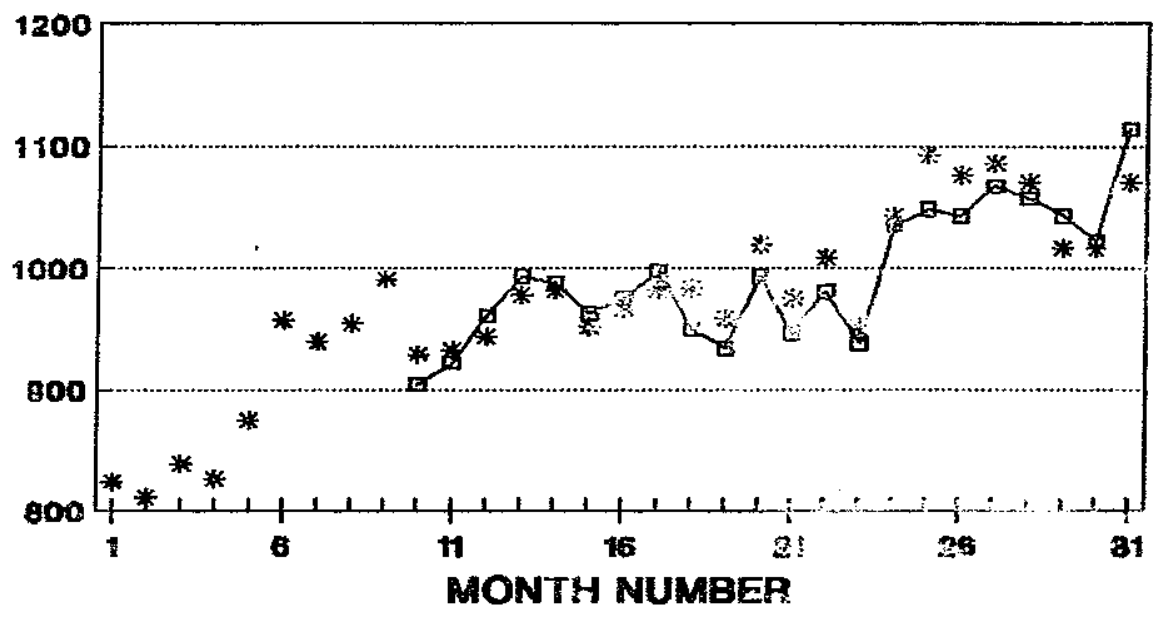

* actual te-forecast co.ouj

Figure 1 : Fast hem with fairly constant demand.

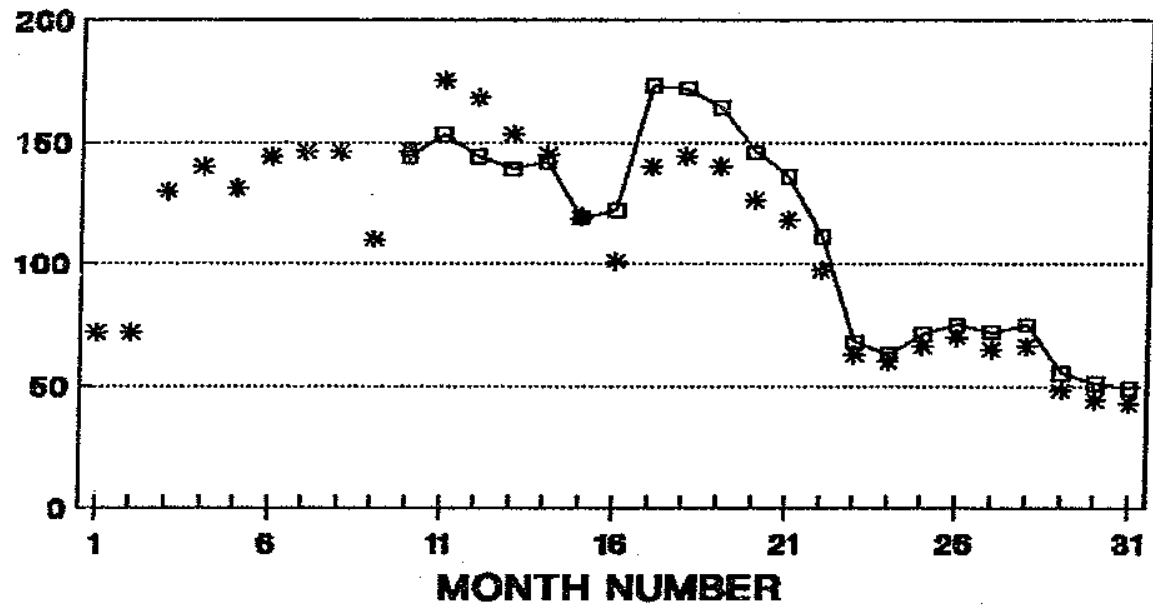

* actual a-Forecast (0.02)

Figure 2 : Fast item with declining demand. 


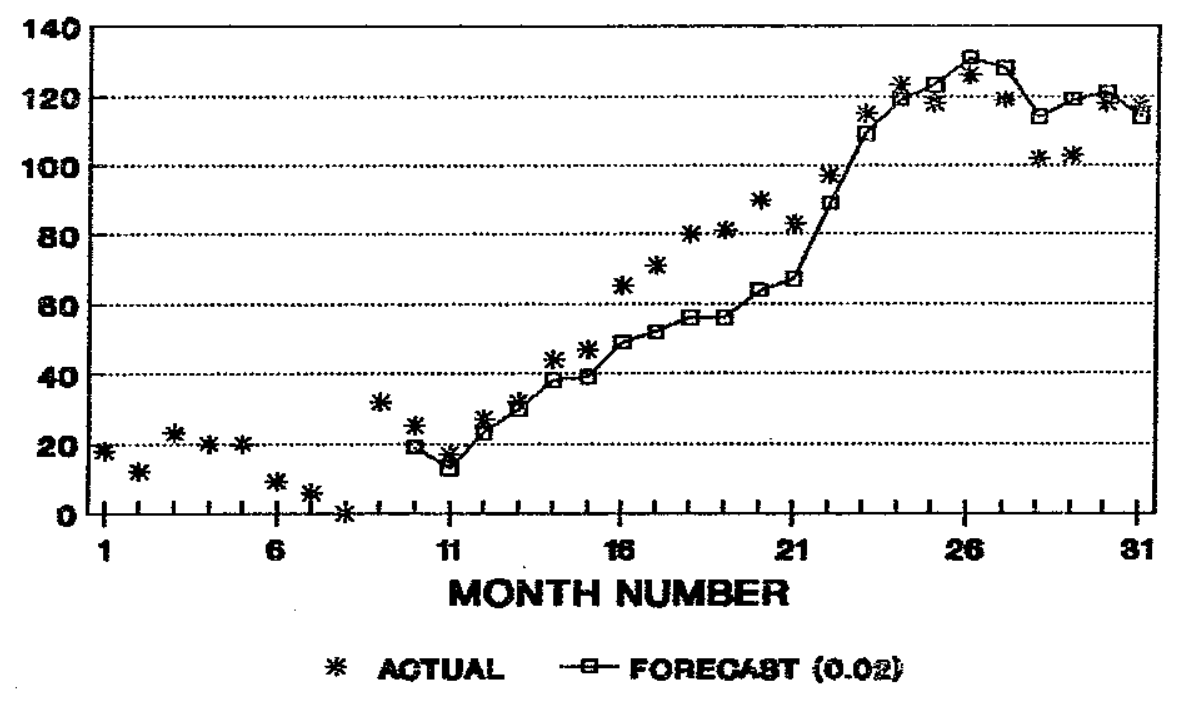

Figure 3 : Fast item with increasing demand.

\section{CONCLUSIONS}

\subsection{WEAKNESSES IN THE METHOD OF EVALUATION}

There are a multitude of complicating factors that may adversely affect the results of forecasts such as this.

The way in which experimental analysis is applied.

Initial conditions and values and the time period over which measurements are done may affect the results.

Simplification of the real system to make it manageable for study.

Many assumptions are made in an evaluation of this nature. Ignoring the typical upward trend of spare part prices are one example.

The physical measurement of what constitutes a good forecast.

This is largely a subjective measure and such subjective measures may have quite radical differences in the eventual results.

Strict selection criteria.

Requirements such as quick response and good accuracy based on little history combined with simplicity needless to say eliminates a large number of possible techniques. 
Practical applicability of techniques.

Many forecasting techniaues rely on the specification of smoothing constants. Such constants are normally determined through experimentation which may be easier said than done.

Statistical treatment of results.

Expressing results as averages of averages have in itself a smoothing effect which may hide all kinds of properties in the results.

The kype of data.

Many zero values in the time series creates computationa? problems when evaluating results.

The total cost of the system is not taken into account.

Although a technique is very accurate on average, steadily buying quantities of

"wrong" items may result in excessive stock levels and expen sive stock write offs.

The human factor.

In practice someone referees an order before placing it with a supplier. When forecasting techniques are evaluated this factor is totally ignored.

Experimenting on only a part of the system.

For the purposes of evaluation all irregular items were discarded and only 832 out of a total quantity of 7,000 were used for the evaluation. The discarded items are typically the problems when forecasts are made in practice.

Suboptimal classification method.

Although the stock classification method may be good for inventory management and reporting purposes, it may not necessarily be optimal for evaluation a forecast.

\subsection{CONCLUSIONS}

In spite of the many potential pitfalls good results were derived with the evaluation. It was evident that Brown's method came out on top for the purposes of forecasting spare part demand. The reasons are as follows :

- It consistently gave the highest acceptable percentage forecast out of all methods evaluated.

- It gave fairly stable acceptable percentage forecasts and the choice of the smoothing constant did not seem too sensitive.

- The range of results were lower than for other techniques.

- It consistently gave the lowest cost variance percentage of all methods evaluated.

- The range of cost variance results was somewhat high but reasonable when compared to other techniques. 
- The combination of both measures provided better results that any other technique for the right choice of smoothing constant.

- It gave good results for all categories of stock items.

- The technique requires very little demand history to provide good results.

- The technique seems to be quite acceptable in practice because it is easily understood.

\subsection{PRACTICAL VALUE OF RESULtS}

The technique is easy to implement in practice and is currently being tested at a pilot site. It is too early to see whether it will give significantly better results that existing methods in use.

In terms of using the technique, the following two strategies can be proposed:

For an optimal acceptable percentage the smoothing constant must be between 0.02 and 0.06 .

For an optimal cost variance percentage a smoothing constant between 0.02 and 0.05 will be best.

A value of 0.03 is currently being used for testing.

Most of us who have worked in the field have had to learn the hard way that techniques accomplish nothing. They are just tools for people."

Oliver W Wight

\section{REFERENCES}

1. Adam, E. E., Individual Item Forecasting Model Evaluation, Decision Sciences, Volume 4 (1973), pp 458-470.

2. Brown, Robert G., STATISTICAL FORECASTING FOR INVENTORY CONTROL, McGraw-Hill, New York, 1959.

3. Brown, Robert G., SMOOTHING, FORECASTING AND PREDICTION OF DISCRETE TIME SERIES, Prentice-Hall, Englewood Cliffs, N. J., 1963.

4. Dalrymple, D. J. and B. E. King, Selecting Parameters for Short-term Forecasting Techniques, Decision Sciences, Volume 12 (1981), pp 661-669.

5. Fortuin, L., The All-time Requirements of Spare Parts for Senvice After Sales Theoretical Analysis and Practical Results, International Journal of Operations and Production Management, Volume 1, pp 59-70, 1980.

6. Gardner, E. S. and Dannenbring, D. G., Forecasting with Exponential Smoothing : Some Guidelines for Model Selection, Decision Sciences, Volume 11 (1980), pp 370-383. 
7. Groff, G.K., Empirical Comparison of Models for Short-range Forecasting, Management Science, Volume 20, Number 1, September 1973, pp 22-31.

8. Makridakis, S., Wheelwright, S. C., McGee, V. E, FORECASTING : Methods and Applications, John Wiley \& Sons, New York, 1983.

9. Moore, J. R., Jr., Forecasting and Scheduling for Past-model Replacement Parts, Management Science, Volume 18, pp B200-B213, 1971.

10. Smith, Bernard T., FOCUS FORECASTING, Oliver Wight Limited Publications, Inc., Essex Junction, VT 05452, 1984.

11. Tersine, R. J. and Toelle, R. A., Optimal Stock Levels for Excess Inventory Items, Journal of Operations Management, Volume 4, pp 245-258, 1984.

-oOo- 